\title{
Process Optimization of Ultrasonic-Assisted Extraction of Arabinogalactan from Dihydroquercetin Extracted Residues by Response Surface Methodology and Evaluation of Its Antioxidant Activity
}

\author{
Zaizhi Liu, Chunhui Ma, Lei Yang, and Yuangang Zu \\ Key Laboratory of Forest Plant Ecology, Ministry of Education, Northeast Forestry University, Harbin 150040, China \\ Correspondence should be addressed to Lei Yang; lyld312@126.com
}

Received 29 May 2013; Revised 28 July 2013; Accepted 3 September 2013

Academic Editor: Antonia Chiou

Copyright (C) 2013 Zaizhi Liu et al. This is an open access article distributed under the Creative Commons Attribution License, which permits unrestricted use, distribution, and reproduction in any medium, provided the original work is properly cited.

Ultrasound was used for the extraction of larch arabinogalactan from Larix gmelinii. The optimal conditions for ultrasound extraction were determined by response surface methodology. Specifically, the Box-Behnken design was employed to evaluate the effects of three independent variables: ultrasound time, temperature, and liquid-solid ratio. The highest arabinogalactan yield $(11.18 \%)$ was obtained under the optimal extraction condition (extraction temperature $41.5^{\circ} \mathrm{C}$, extraction time $24.3 \mathrm{~min}$, and liquidsolid ratio $40 \mathrm{~mL} / \mathrm{g}$ ). In addition, the antioxidant activity of arabinogalactan that was extracted from dihydroquercetin extraction residues exhibited a moderate and concentration-dependent hydroxyl radical-scavenging capacity, ferric-reducing power, and reducing power. The wood material was characterized before and after processing by scanning electron microscopy and Fouriertransform infrared spectroscopy.

\section{Introduction}

Larix gmelinii is a medium-sized, deciduous coniferous tree primarily distributed in Greater and Lesser Khingan of China, north Sakhalin, and east Siberia [1]. Its rigidness, straight grain, and stability have made L. gmelinii a widely used wood for furniture and buildings. As a result, large quantities of logging slashes (stumps, branches, roots), bucking residues, and processing residues (sawdust, crushed veneer, wood core, shavings) are produced every year as side products. The extensive environmental impact has created a focus on the comprehensive use of the L. gmelinii resource. It has been reported that it contains valuable bioflavonoids such as dihydroquercetin (taxifolin) [2] and polymeric procyanidins [3]. These bioactive components in L. gmelinii residues could be extracted for an economical utilization. In the conventional process of dihydroquercetin production, ethanol has been used as the extraction solvent, and large amounts of remainder residues have been produced and discarded [4-6].
Arabinogalactan is a polysaccharide in the woody tissue of the Dahurian larch (L. gmelinii) and the Siberian larch (L. sibirica). Arabinogalactan content in those trees is at least $10 \%$ [7]. The arabinogalactan macromolecule has a galactan core with $D$-galactopyranose linked by $\beta-1,3-O-$ glucosides [8]. Larch arabinogalactan is a highly branched polysaccharide that consists of $\beta$-D-galactopyranose, $\alpha-L$ arabinofuranose, and $\beta$ - $L$-arabinopyranose residues [9]. Arabinogalactan is generally recognized in the United States as safe for consumption as a soluble and nonviscous dietary fiber and is commercially used as a dietary supplement [10]. It is currently being used in food and beverages and has been accepted by consumers [11]. Furthermore, numerous pharmacological effects of larch arabinogalactan have been reported such as gastroprotection [8], membrane-tropic [12], anti-inflammation [8], and immunity enhancements [13-15]. Interest is also fueled by its unique properties that make it possible to be used as a biomacromolecular drug carrier that prolongs the action of pharmaceuticals, and it can 
conjugate with drugs to improve their bioaccessibility [1618]. Arabinogalactan can be converted into valuable products through a sugar platform by way of two processes: hydrolysis to monomers and catalytic transformation of the sugars into bio-based products. The monomers of $L$-arabinose and $D$ galactose have been used as specialty sugars in the food, pharmaceutical, and cosmetic industries $[19,20]$. Thus, in summary, arabinogalactan has been recognized as a multipurpose natural product with great economic potential and environmental impact.

The preliminary experiments undertaken in the present study have shown that the extract residues in the production of dihydroquercetin contain large amounts of arabinogalactan. As discussed above, arabinogalactan has a remarkable range of applications, and discarding it would be a waste of valuable plant resources. Hence, it is important to optimize the extraction process for arabinogalactan separation from the dihydroquercetin extract residues.

Many methods have been developed for the separation of polysaccharides, such as maceration extraction and heat reflux extraction. However, these separation methods are inefficient which result in a low recovery of the product, time-consuming, solvent-consuming, energy-consuming, and even bad environment conditions. Thus, an alternative extraction technique that is simple, rapid, highly efficient, environmentally friendly, and inexpensive is urgently needed. Ultrasound-assisted extraction (UAE) is one way to solve this problem [21-23]. It offers high reproducibility, short extraction times, simple manipulation, low solvent consumption, low temperatures, and low energy input [24-26].

The objective here is to develop an effective and environmentally friendly UAE approach for the extraction of the arabinogalactan from the extract residues in the production of dihydroquercetin. The arabinogalactan yield was optimized using response surface methodology. The microstructures of L. gmelinii samples before and after the extraction were characterized by scanning electron microscopy (SEM) and Fourier-transform infrared spectroscopy (FTIR). Finally, the antioxidant activities of the extracted arabinogalactan were evaluated by its hydroxyl radical-scavenging capacity, its ferric-reducing antioxidant power, and its reducing power.

\section{Experimental}

2.1. Chemicals and Materials. 2,4,6-Tripyridyl-s-triazine (TPTZ) and 6-hydroxy-2,5,7,8-tetramethylchroman-2-carboxylic acid (Trolox), an arabinogalactan standard, and vitamin $\mathrm{C}$ were purchased from Sigma Aldrich (St. Louis, MO, USA). All other solvents and chemicals were of analytical grade from Beijing Chemical Reagents Co. (Beijing, China). Deionized water was purified by a Milli-Q water purification system (Millipore, Billerica, Mass, USA).

A bark-free L. gmelinii stump was provided by Mohe Forestry (Heilongjiang, China). It was dried at ambient temperature for 1 month, ground into a homogeneous powder, and then sieved (60-80 mesh).

Pretreatment processing of the raw material was carried out as follows. The samples were defatted in a reflux extraction apparatus with petroleum ether at $60-90^{\circ} \mathrm{C}$ and soaked twice with $80 \%$ ethanol for $4 \mathrm{~h}$ at room temperature in a slow oscillation water bath to remove soluble components, including free sugars, amino acids, and some phenols (such as dihydroquercetin). The ethanolic mixture was then centrifuged, and the wet larch wood powder was vacuum-dried at $60^{\circ} \mathrm{C}$. The dihydroquercetin residues were stored at room temperature in a desiccator until used. The same batch of the samples was used here in the experiments.

2.2. Ultrasonic-Assisted Extraction Apparatus. For the UAE experiments, a KQ-250DB ultrasonic bath (Kunshan, Jiangsu, China) was used in the extraction step. The bath was a rectangular container $\left(23.5 \times 13.3 \times 10.2 \mathrm{~cm}^{3}\right)$, to which $50 \mathrm{kHz}$ transducers were attached at the bottom. The bath power rating was $250 \mathrm{~W}$ on a scale of $40-100 \%$. An SHBIIIA pump (Zhengzhou, China) and circulating water system maintained a constant temperature.

2.3. Extraction and Quantification of the Arabinogalactan Yield. The pretreated powder $(10.0 \mathrm{~g})$ was immersed in deionized water. The extraction process was performed at different ultrasound temperatures and times and liquid-solid ratios. The optimal extraction conditions were calculated by response surface methodology. The mixture was centrifuged $(4800 \times \mathrm{g}, 10 \mathrm{~min})$, and the supernatant was separated from the insoluble residue with a four-layer filter cloth. The extracts were precipitated by the addition of ethanol to a final concentration of $75 \%(\mathrm{v} / \mathrm{v})$ and incubated overnight at $4^{\circ} \mathrm{C}$. The precipitates were then collected by centrifugation $(8000 \times \mathrm{g}$ for $30 \mathrm{~min}$, at $20^{\circ} \mathrm{C}$ ), washed three times with dehydrated alcohol, and freeze-dried. A phenol/sulfuric acid procedure [27] was performed to determine the polysaccharide content using glucose as a standard. The polysaccharide yield was expressed as gram of glucose equivalent (GE) per 100 grams of L. gmelinii dihydroquercetin-extracted residues on a dry weight basis (DWB). All tests (except for the five replicates at the center of the Box-Behnken design) were performed in triplicate and averaged.

2.4. Box-Behnken Design. The Design Expert (Version 8.0, Stat-Ease Inc., Minneapolis, MN, USA) software was used for experimental design, data analysis, and model building. A Box-Behnken design was used to determine the response pattern and then to establish a model. The liquid-solid ratio and the ultrasound time and temperature were chosen as the key variables based on the results of preliminary experiments and were designated $X_{1}, X_{2}$, and $X_{3}$, respectively, as shown in Table 1 . Five replicates at the center of the design were used for the estimation of a pure-error sum of squares. Experiments were randomized to maximize the effects of unexplained variability, due to extraneous factors, in the observed responses. A quadratic equation was used for this model as follows:

$$
Y=\beta_{0}+\sum_{i=1}^{3} \beta_{i} X_{i}+\sum_{i=1}^{3} \beta_{i i} X_{i}^{2}+\sum_{i=1}^{2} \sum_{j=i+1}^{3} \beta_{i j} X_{i} X_{j},
$$

where $Y$ is the estimated response, $\beta_{0}, \beta_{i}, \beta_{i i}$, and $\beta_{i j}$ are the regression coefficients for the intercept, linearity, square, 
TABle 1: The Box-Behnken design matrix to screen important variables, and the extraction yields of crude polysaccharides.

\begin{tabular}{|c|c|c|c|c|c|}
\hline Run & $\begin{array}{c}\text { (Coded value) } \\
\text { Factor } X_{1} \\
\text { Liquid-solid ratio } \\
(\mathrm{mL} / \mathrm{g})\end{array}$ & $\begin{array}{c}\text { (Coded value) } \\
\text { Factor } X_{2} \\
\text { Ultrasound irradiation } \\
\text { time }(\mathrm{min})\end{array}$ & $\begin{array}{c}\text { (Coded value) } \\
\text { Factor } X_{3} \\
\text { Extraction } \\
\text { temperature }\left({ }^{\circ} \mathrm{C}\right) \\
\end{array}$ & $\begin{array}{c}\text { Predicted response } \\
\text { Yield (\%) }\end{array}$ & $\begin{array}{c}\text { Actual response } \\
\text { Yield (\%) }\end{array}$ \\
\hline 1 & (1) 40 & (0) 30 & $(-1) 40$ & 11.02 & 11.16 \\
\hline 2 & (0) 30 & (0) 30 & (0) 50 & 9.75 & 9.88 \\
\hline 3 & $(-1) 20$ & (-1) 20 & (0) 50 & 8.84 & 9.04 \\
\hline 4 & (1) 40 & (1) 40 & (0) 50 & 9.73 & 9.53 \\
\hline 5 & $(-1) 20$ & (0) 30 & (1) 60 & 9.85 & 9.71 \\
\hline 6 & (-1) 20 & (1) 40 & (0) 50 & 9.85 & 10.14 \\
\hline 7 & (1) 40 & $(-1) 20$ & (0) 50 & 10.88 & 10.59 \\
\hline 8 & (0) 30 & $(-1) 20$ & $(-1) 40$ & 10.34 & 10.49 \\
\hline 9 & (0) 30 & (-1) 20 & (1) 60 & 8.65 & 8.60 \\
\hline 10 & (0) 30 & (0) 30 & (0) 50 & 9.75 & 9.19 \\
\hline 11 & (0) 30 & (0) 30 & (0) 50 & 9.75 & 9.78 \\
\hline 12 & (0) 30 & (1) 40 & (1) 60 & 10.06 & 9.91 \\
\hline 13 & (0) 30 & (1) 40 & $(-1) 40$ & 8.79 & 8.84 \\
\hline 14 & (0) 30 & (0) 30 & (0) 50 & 9.75 & 9.90 \\
\hline 15 & (-1) 20 & (0) 30 & (-1) 40 & 9.45 & 9.10 \\
\hline 16 & (0) 30 & (0) 30 & (0) 50 & 9.75 & 9.98 \\
\hline 17 & (1) 40 & (0) 30 & (1) 60 & 10.20 & 10.54 \\
\hline
\end{tabular}

and interaction, respectively, and $X_{1}, X_{2}$, and $X_{3}$ are the independent variables.

2.5. Control Experiment. Hot water extraction (HWE) was employed as a control experiment for the arabinogalactan preparation. The pretreated powders $(10.0 \mathrm{~g})$ were placed in a flask, extracted with $100 \mathrm{~mL}$ deionized water for $2 \mathrm{~h}$, and then centrifuged $(4800 \times \mathrm{g}, 10 \mathrm{~min})$. The subsequent extraction procedures for the arabinogalactan were the same as discussed above. All tests were performed in triplicate and averaged.

2.6. Assay for Hydroxyl Radical-Scavenging Capacity. The - $\mathrm{OH}$ radical-scavenging capacity of the arabinogalactan was evaluated according to the reaction of sodium salicylate with residual hydroxyl radical, and the assay was performed according to a reported procedure [28], with few modifications. Hydroxyl radicals were generated by the Fenton reaction of $\mathrm{FeSO}_{4}$ and $\mathrm{H}_{2} \mathrm{O}_{2}$. The reaction mixture consisted of $0.5 \mathrm{~mL} \mathrm{mM} \mathrm{FeSO}_{4}, 0.8 \mathrm{~mL} 6 \mathrm{mM} \mathrm{H}_{2} \mathrm{O}_{2}, 0.5 \mathrm{~mL}$ deionized water, $1.0 \mathrm{~mL}$ of various concentrations of the extracts, and $0.2 \mathrm{~mL} 20 \mathrm{mM}$ sodium salicylate. The total mixture $(3.0 \mathrm{~mL})$ was kept at $37^{\circ} \mathrm{C}$ for $1 \mathrm{~h}$, after which the absorbance at $562 \mathrm{~nm}$ was recorded. Vitamin $\mathrm{C}$ was used as a reference. All tests were performed in triplicate and averaged. The scavenging activity was calculated using the following equation:

Scavenging (or inhibition) rate $(\%)=\frac{A_{1}-A_{2}}{A_{0}} \times 100$, where $A_{0}$ is the absorbance of the control (without the extracts), $A_{1}$ is the absorbance of the extracted sample, and $A_{2}$ is the absorbance without sodium salicylate.

2.7. Assay for Ferric-Reducing Antioxidant Power (FRAP). The FRAP assay was carried out according to the procedure of Li et al. [29] with slight modifications. Briefly, the FRAP reagent was freshly prepared by mixing $25 \mathrm{~mL} 300 \mathrm{mM}$ of acetate buffer ( $\mathrm{pH} 3.6), 2.5 \mathrm{~mL} 10 \mathrm{mM}$ of TPTZ solution, and $2.5 \mathrm{~mL} 20 \mathrm{mM}$ of $\mathrm{FeCl}_{3}$ solution. It was stored at $37^{\circ} \mathrm{C}$ for $30 \mathrm{~min}$ prior to use. The arabinogalactan was dissolved in deionized water at concentrations of 5, 10, 15, 20, and $25 \mathrm{mg} / \mathrm{mL}$. A $0.15 \mathrm{~mL}$ sample of each solution was mixed separately with $2.85 \mathrm{~mL}$ of FRAP reagent and then kept for $30 \mathrm{~min}$ in the dark. The absorbance of the reaction/extract solution was measured at $593 \mathrm{~nm}$. A standard curve was constructed using 37.5 to $600 \mu \mathrm{M}$ Trolox. The antioxidant efficiencies of the samples were calculated with reference to the Trolox reaction signal at a known concentration. The results were corrected for dilution and expressed as $\mu \mathrm{mol}$ Trolox equivalents (TE)/g. Vitamin $\mathrm{C}$ was used as a positive control. The assay was performed in triplicate and averaged.

2.8. Assay for Reducing Power. The reducing power of the arabinogalactan was determined as described by Kumar et al., Katekhaye and Kale, and Negi et al. [30-32], with slight modifications. Briefly, $1.0 \mathrm{~mL}$ of each sample solution (12.5, $15,17.5,20,22.5$, and $25 \mathrm{mg} / \mathrm{mL}$ ) was mixed separately with $2.5 \mathrm{~mL}$ of $0.2 \mathrm{M}$ phosphate buffer ( $\mathrm{pH} 6.6$ ) and $2.5 \mathrm{~mL}$ of $1 \%(\mathrm{w} / \mathrm{v})$ potassium ferricyanide. The mixture was placed in a water bath at $50^{\circ} \mathrm{C}$ for $20 \mathrm{~min}$. Then $2.5 \mathrm{~mL}$ of $10 \%$ 
$(\mathrm{w} / \mathrm{v})$ trichloroacetic acid was added, and the mixture was centrifuged at $3000 \times \mathrm{g}$ for $10 \mathrm{~min}$. The supernatant $(2.5 \mathrm{~mL})$ was combined with $2.5 \mathrm{~mL}$ of deionized water and $0.5 \mathrm{~mL}$ of $0.1 \%$ ferric chloride, and the absorbance was measured at $700 \mathrm{~nm}$. Vitamin $\mathrm{C}$ was used as a positive control, where $1.0 \mathrm{~mL}$ of $1 \mathrm{mg} / \mathrm{mL}$ vitamin $\mathrm{C}$ was pipetted to a $10 \mathrm{~mL}$ volumetric flask, diluted to volume, and then mixed as the mother liquor. The reducing power of the vitamin $\mathrm{C}$ was evaluated by double diluting the mother liquor to make sure that the absorbance had Beer-Lambert linearity. All tests were performed in triplicate and averaged.

2.9. Scanning Electron Microscopy. Characterization of the L. gmelinii wood powder morphology before and after processing was performed with a scanning electron microscope (SEM, Quanta 200, FEI, USA). Samples were sputter-coated with a thin layer of gold-palladium $(5-10 \mathrm{~nm} ; 10 \mathrm{~mA} ; 30 \mathrm{~s})$ at room temperature before imaging.

2.10. Fourier-Transform Infrared Spectroscopy (FTIR). Arabinogalactan and the unprocessed and processed L. gmelinii wood powder samples were separately diluted and ground with spectroscopic grade $\mathrm{KBr}$ mixing powder at $1 \%$ concentrations and pressed at a load of 5 tons for $2 \mathrm{~min}$ to obtain self-supporting disks for FTIR spectroscopy. Spectra were obtained with a MAGNA-IR560 E.S.P (Nicolet, USA) and recorded over the midinfrared range of $4000-400 \mathrm{~cm}^{-1}$ at a resolution of $5 \mathrm{~cm}^{-1}$.

2.11. Statistical Analysis. All data (including the sections of $2.3,2.5,2.6,2.7$, and 2.8, except for the five replicates at the center of the Box-Behnken design) were the mean of three replicate determinations within significance $P<0.05$ after being subjected to an analysis of variance (ANOVA) and processed with SPSS 13.0. The "Design Expert" software version 8.0 (Stat-Ease, Minneapolis, USA) was employed for the regression analysis and the graphical optimization.

\section{Results and Discussion}

3.1. Effects of Ultrasound Time, Temperature, and LiquidSolid Ratio on the Arabinogalactan Yield. Response surface curves were drawn to evaluate the links of the variables and to determine the optimal level of each variable for the maximum response. The optimal values of the selected variables were obtained by solving the regression equation using the "Design Expert" software version 8.0. The 3D response surface and the $2 \mathrm{D}$ contour plots were graphical representations of the regression equation. Each contour curve represented an infinitive number of combinations of two test variables, keeping the other factors fixed at the zero level. Figure 1(a) shows that the yield of the arabinogalactan increased with increasing liquid-solid ratio and decreasing extraction time. Figure 1(b) shows the effect of liquid-solid ratio, extraction temperature, and their reciprocal interaction on the extraction yield. When the liquid-solid ratio was fixed at $40 \mathrm{~mL} / \mathrm{g}$, the arabinogalactan yield decreased with increasing extraction temperatures over the range $40-60^{\circ} \mathrm{C}$.
When the extraction temperature was fixed at $40^{\circ} \mathrm{C}$, there was a quadratic effect on the yield with increasing liquidsolid ratios over the range $20-40 \mathrm{~mL} / \mathrm{g}$. Likewise, as is shown in Figure 1(c), the extraction time had a negative effect on the yield when the extraction temperature was fixed at $40^{\circ} \mathrm{C}$. There was a significant effect on the yield with increasing extraction temperature and increasing extraction time.

The optimum extraction conditions $\left(X_{1}=40 \mathrm{~mL} / \mathrm{g}\right.$, $X_{2}=24.3 \mathrm{~min}$, and $X_{3}=41.5^{\circ} \mathrm{C}$ ) for the arabinogalactan yield were estimated using the model equation and by solving the regression equation. The theoretical arabinogalactan yield that was predicted for the above conditions was $11.18 \%$.

3.2. Model Building and Statistical Analysis. The data obtained from all the experiments are summarized in Table 1. There were a total of 17 runs for optimizing the three individual parameters that determined the arabinogalactan yield. The values of responses (i.e., the arabinogalactan yields) under different experimental combinations of the coded variables are given in Table 1 . The percentage yields ranged from $8.60 \%$ to $11.16 \%$.

The design variables in coded units are given in Table 1 along with the predicted and experimental values of the response. Each data point was the average of three sets of experiments, whereas the predicted values of the responses were obtained from the quadratic model fitting technique by the software.

By applying multiple regression analysis on the experimental data, the response variable and the test variables were related by the second-order polynomial equation as follows:

$$
\text { Yield \%= } \begin{array}{rl}
\% & 11.49+0.14 X_{1}-0.035 X_{2}-0.15 X_{3} \\
& -5.4 \times 10^{-3} X_{1} X_{2}+7.4 \times 10^{-3} X_{2} X_{3} .
\end{array}
$$

The significance of each coefficient was checked using the $F$-test and the $P$ value (Table 2 ). The $P$ value was used to check the significance of each coefficient, and it also indicated the interaction strength between each independent variable. The ANOVA of the quadratic regression model demonstrated that the model was significant, which was evident from the $F$-test low probability value $(P=0.0204)$. The $5.20 \mathrm{Model}$ $F$-value also implies that the model was significant, since there was only a $2.04 \%$ chance that a value this large could occur because of noise. The "Lack of fit F-value" of 1.89 was not significant relative to the pure error. There was a $27.30 \%$ chance that a "Lack of fit F-value" this large could occur because of noise. Thus, the nonsignificant lack of fit showed the model was suitable for data analysis. The regression coefficients and the corresponding $P$ values are also shown in Table 2. Values of "Prob $>F$ " less than 0.0500 indicate that the $X_{1}, X_{1} X_{2}, X_{2} X_{3}$ model terms were significant. Values greater than 0.1000 indicate model terms that were not significant, such as $X_{2}, X_{3}, X_{1} X_{3}, X_{1}^{2}, X_{2}^{2}$, and $X_{3}^{2}$. The coefficient of determination $\left(R^{2}=0.8700\right)$, the adjusted coefficient of determination $\left(R_{\text {Adj }}^{2}=0.7028\right)$, and the coefficient of variation (C.V. $=3.83 \%)$ are shown in Table 2. The $R_{\text {Adj }}^{2}$ is a modification of the $R^{2}$ that adjusts for the number of 


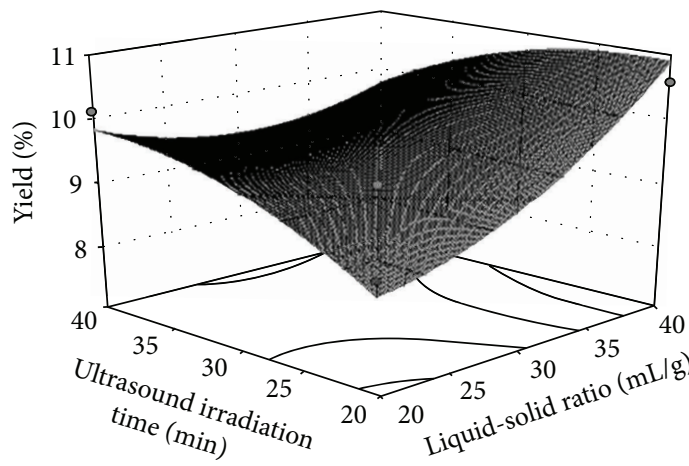

Extraction temperature $\left({ }^{\circ} \mathrm{C}\right)=50$

(a)

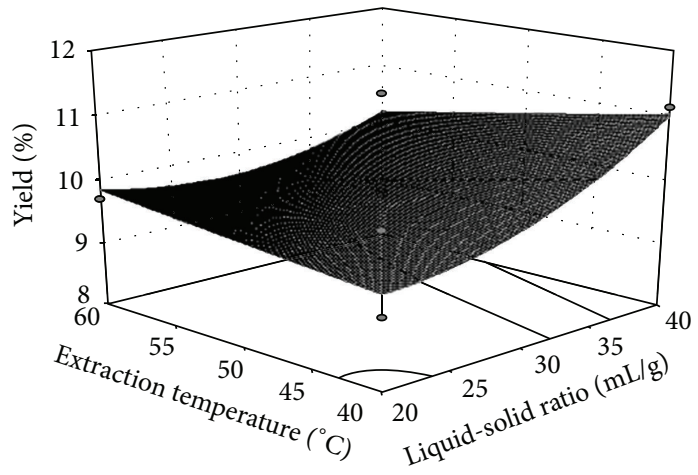

Ultrasound irradiation time $(\min )=30$

(b)

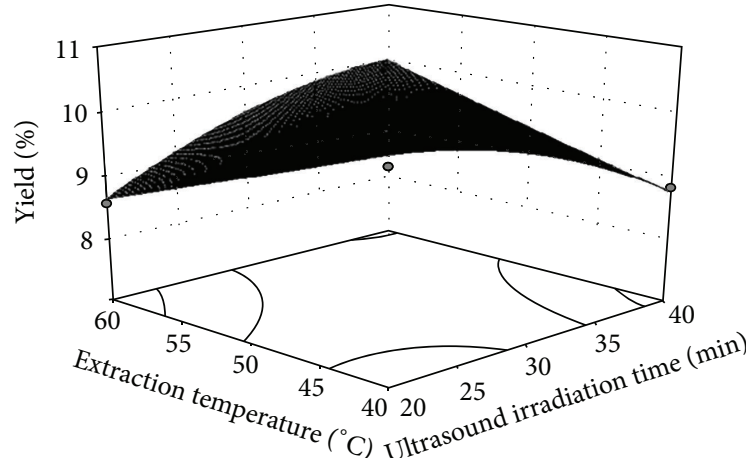

Liquid-solid ratio $(\mathrm{mL} / \mathrm{g})=30$

(c)

FIGURE 1: Response surface plots showing the effects of variables on the UAE extraction yield of larch arabinogalactan. (a) Interaction of liquid-solid ratio and irradiation time. (b) Interaction of liquid-solid ratio and extraction temperature. (c) Interaction of irradiation time and extraction temperature.

TABLE 2: Test of significance for regression coefficient and the credibility analysis ${ }^{\mathrm{a}}$.

\begin{tabular}{|c|c|c|c|c|c|c|}
\hline Source & Sum of squares & Regression coefficient & Degree of freedom & Mean square & $F$-value & $P$-value \\
\hline Model $^{\text {b }}$ & 6.57 & 9.75 & 9 & 0.73 & 5.20 & 0.0204 \\
\hline$X_{1}$ & 1.83 & 0.48 & 1 & 1.83 & 13.07 & 0.0086 \\
\hline$X_{2}$ & 0.01 & -0.04 & 1 & 0.01 & 0.08 & 0.7852 \\
\hline$X_{3}$ & 0.09 & -0.10 & 1 & 0.09 & 0.61 & 0.4591 \\
\hline$X_{1} X_{2}$ & 1.17 & -0.54 & 1 & 1.17 & 8.31 & 0.0235 \\
\hline$X_{1} X_{3}$ & 0.38 & -0.31 & 1 & 0.38 & 2.70 & 0.1446 \\
\hline$X_{2} X_{3}$ & 2.19 & 0.74 & 1 & 2.19 & 15.61 & 0.0055 \\
\hline$X_{12}$ & 0.59 & 0.37 & 1 & 0.59 & 4.18 & 0.0801 \\
\hline$X_{22}$ & 0.36 & 0.29 & 1 & 0.36 & 2.60 & 0.1510 \\
\hline$X_{32}$ & 0.00 & 0.00 & 1 & 0.00 & 0.00 & 0.9652 \\
\hline Residual & 0.98 & & 7 & 0.14 & & \\
\hline Lack of fit & 0.58 & & 3 & 0.19 & 1.89 & 0.2730 \\
\hline Pure error & 0.41 & & 4 & 0.10 & & \\
\hline Cor total & 7.55 & & 16 & & & \\
\hline Std. dev. & Mean & C.V. \% & $R^{2}$ & Adjust $R^{2}$ & Predicted $R^{2}$ & Adequacy precision \\
\hline 0.37 & 9.79 & 9.84 & 0.8700 & 0.7028 & -0.3029 & 8.2281 \\
\hline
\end{tabular}

${ }^{\mathrm{a}}$ The results were obtained with the Design Expert 8.0 software.

${ }^{\mathrm{b}} X_{1}$ is liquid-solid ratio $(\mathrm{mL} / \mathrm{g}) . X_{2}$ is ultrasound irradiation time $(\mathrm{min}) . X_{3}$ is extraction temperature $\left({ }^{\circ} \mathrm{C}\right)$. 
predictors in the model. The $R_{\text {Adj }}^{2}$ is usually used to compare the explanatory power of regression models which contain different numbers of predictors. It increases only if the new term improves the model more than expected by chance. The $R_{\text {Adj }}^{2}$ decreases when a predictor improves the model by less than expected by chance. It is always lower than the $R^{2}$. In this work, the $R_{\text {Adj }}^{2}(0.7028)$ is lower than the $R^{2}(0.8700)$, which is an indication that the regression equation is overfitted to the sample and of limited generalizability. "Adeq Precision" measured the signal-to-noise ratio. A ratio greater than 4 was desirable, thus a ratio of 8.228 indicated an adequate signal. This model can be used to navigate the design space.

3.3. Validation of the Model and Control Experiment. The optimum extraction conditions were applied to three independent duplications of arabinogalactan extraction to prove the model prediction. The mean extraction arabinogalactan yield was $10.63 \pm 0.67 \%$, which corresponds well with the predicted conditions of the model equation. Thus, the response model was suitable for the optimization. The reference method was HWE, which had an arabinogalactan yield from the dihydroquercetin extraction residues of only $9.53 \pm$ $0.84 \%$.

Relative to HWE, the application of UAE improved the arabinogalactan yield. Using a short UAE extraction time (24 min), the arabinogalactan yield increased to $11.18 \%$, which represented an increase of 5.17\% over that of HWE (9.53\%). Moreover, the extraction time decreased by a factor of 5 . The higher efficiency of the UAE method can be explained by the mechanical disruption of the cell walls of the samples, resulting in a significantly increased accessibility and extractability of the arabinogalactan. Therefore, UAE is an efficient technique for the extraction of arabinogalactan from the dihydroquercetin extraction residues, because it resulted in a maximum extraction yield in a shorter extraction time. Compared with HWE, UAE saved time and energy and gave a higher extraction yield. UAE is a new extraction technology which has been developed rapidly in recent years. The acoustic cavitation in UAE causes disruption of the cell walls, reduction of the particle size and enhancement on contact between solvents and targeted compounds. UAE is usually used in the process of extracting bioactive substances from plant material. During the past years, as there is not large industrialization equipment, it is much difficult to be applied to actual production. In recent years, a variety of special industrial extraction equipment has appeared, such as circulation ultrasonic extractor and ultrasonic continuous countercurrent extractor, which makes the application of UAE in the large-scale extraction procedures become a reality. Compared to the UAE equipment adopted in this work, though they have some differences, but their principles are the same. Thus, it could be an effective and advisable technique for large-scale extraction of Larix arabinogalactan and even other sources of arabinogalactans in food industry.

3.4. Hydroxyl Radical-Scavenging Ability. The scavenging capacity of arabinogalactan extracts for hydroxyl radical inhibition was characterized by the 2-deoxyribose oxidation method. As shown in Figure 2(a), the results indicated that inhibition by the arabinogalactan $(25 \mathrm{mg} / \mathrm{mL})$ reduced the hydroxyl radical-scavenging activity to $37.42 \%$. In contrast, the inhibition by vitamin $\mathrm{C}(0.5 \mathrm{mg} / \mathrm{mL})$ indicated a higher hydroxyl radical-scavenging activity $(66.30 \%)$ by a lower concentration of antioxidant.

3.5. Ferric-Reducing Antioxidant Power. The FRAP assay is generally used to measure the reduction of ferric ion to ferrous ion in the presence of antioxidants and is commonly used for the analysis of total antioxidant activity of the extracts. Thus, it measures the antioxidant activity of any substance in the reaction medium as an equivalent reducing power. A high FRAP value certifies high antioxidant activity. The antioxidant activities of arabinogalactan from the dihydroquercetin extraction residues are shown in Figure 2(b). The arabinogalactan displayed lower FRAP antioxidant activity relative to that of vitamin C. However, the data indicate that the FRAP activity of arabinogalactan increased with sample concentration increasing.

3.6. Reducing Power. In this experiment, the yellow color of the test solution changes to green depending on the reducing power of the test specimen; that is, eductants in the solution cause the conversion of the ferricyanide complex to the ferrous form. The ferrous form can be monitored by the absorbance at $700 \mathrm{~nm}$. Figure 2(c) displays the reducing power of arabinogalactan and vitamin $\mathrm{C}$. The reducing power of arabinogalactan was dose-dependent. As expected, its reducing power was much less than that of vitamin $C$, which is known to be a strong reducing agent. The reducing power of vitamin $C$ was 0.876 at a dose of $0.05 \mathrm{mg} / \mathrm{mL}$, while that of arabinogalactan was 0.182 at a dose of $25 \mathrm{mg} / \mathrm{mL}$. Arabinogalactan can therefore act as an electron donor and can react with free radicals to convert them to more stable forms, and stop radical chain reactions. However, its antioxidant activity is not as strong as that of vitamin C.

The FRAP results correlated well with those found for the hydroxyl radical-scavenging activity assay and the reducing power assay, indicating that the arabinogalactan antioxidant activity was consistent. The antioxidant activity assays indicated that the arabinogalactan is very stable and that this kind of polysaccharide may be used as specialty sugars with potential value for healthy food in food industry.

3.7. Structural Changes after Extraction. FTIR spectroscopy was used to survey changes in the chemical structures of the arabinogalactan before and after extraction by the various methods. FTIR spectra of unprocessed and processed L. gmelinii sample powders are plotted in Figure 3(a). They reveal that the intensities of the absorption bands at $3440,2947,1745,1652,1510,1280$, and $1070 \mathrm{~cm}^{-1}$ did not significantly change following UAE or HWE processing. Thus, they indicate that the chemical composition of the cell wall was unchanged after extraction by either of these methods. 


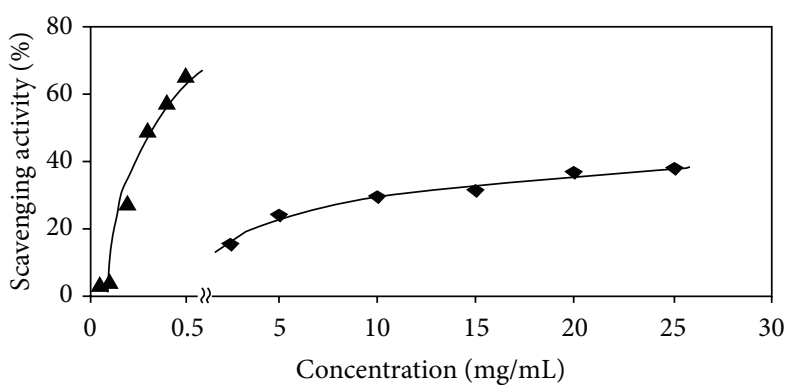

- Arabinogalactan

- Vitamin C

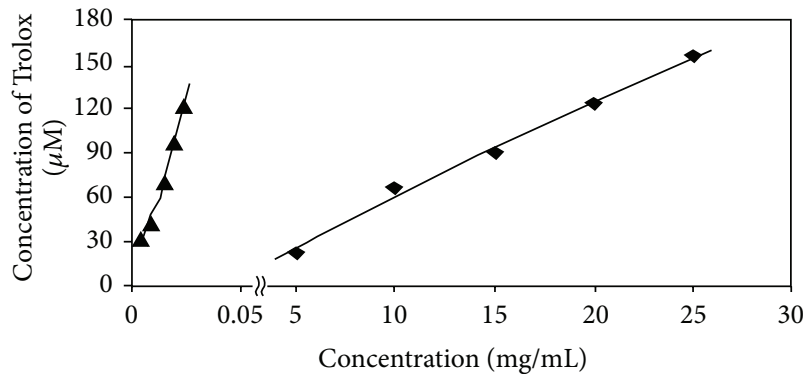

- Arabinogalactan

$\Delta$ Vitamin C

(a)

(b)

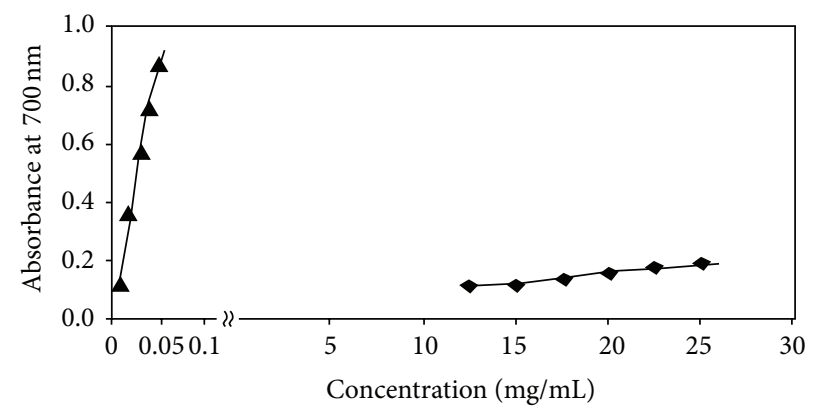

- Arabinogalactan

$\Delta$ Vitamin C

(c)

Figure 2: Antioxidant activities of larch arabinogalactan compared with vitamin C. (a) Hydroxyl radical-scavenging capacity. (b) Ferricreducing antioxidant power. (c) Reducing power.

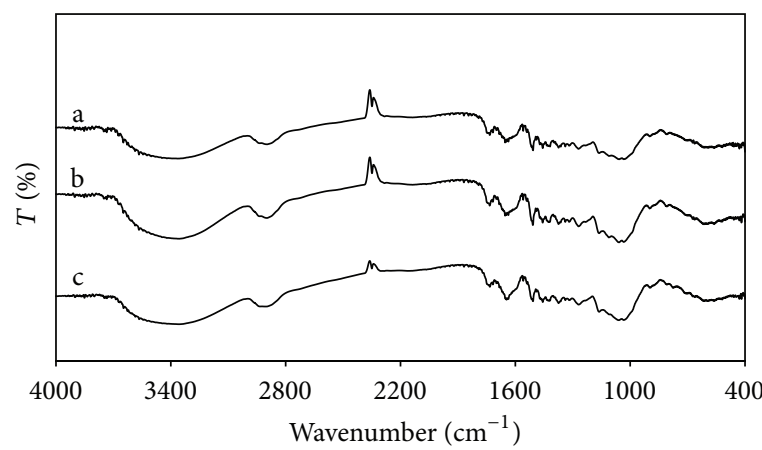

(a)

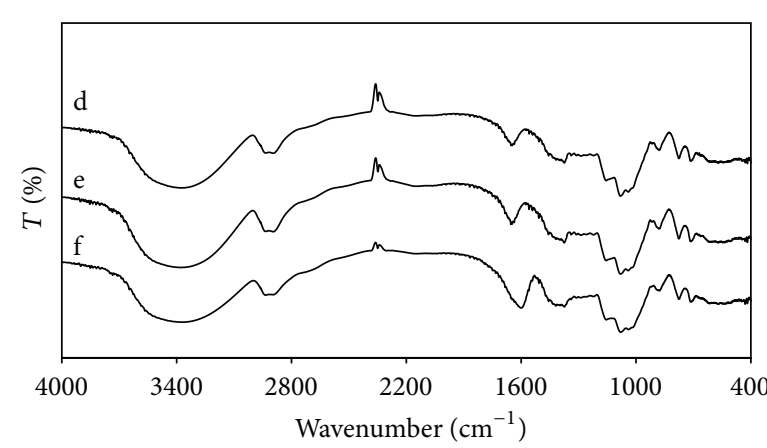

(b)

FIGURE 3: FTIR spectra. (a) samples of L. gmelinii: a-HWE processed. b-UAE processed. c-Unprocessed. (b) Arabinogalactan: d-HWE. e-UAE. f-Standard.

The FTIR spectrum of arabinogalactan is shown in Figure 3(b). The absorption bands at $3600-3200 \mathrm{~cm}^{-1}, 3000-$ $2800 \mathrm{~cm}^{-1}, 1800-1600 \mathrm{~cm}^{-1}$, and $1200-1000 \mathrm{~cm}^{-1}$ are characteristic of arabinogalactan. The broad intense peak at $3410 \mathrm{~cm}^{-1}$ is a hydroxyl stretch and the peaks at $2950 \mathrm{~cm}^{-1}$, $2900 \mathrm{~cm}^{-1}, 1400 \mathrm{~cm}^{-1}$, and $1170 \mathrm{~cm}^{-1}$ correspond to weak $\mathrm{C}-\mathrm{H}$ modes. The peak at $1660 \mathrm{~cm}^{-1}$ is attributed to an N$\mathrm{H}$ vibration or a $\mathrm{C}=\mathrm{O}$ asymmetric vibration of a carboxyl group, which is also a characteristic of arabinogalactan. FTIR spectra of arabinogalactan from UAE, HWE, and an arabinogalactan standard are plotted in Figure 3(b). The similar spectra indicate that the chemical structures and purities of the arabinogalactan standard were not significantly different from arabinogalactan extracted by the two methods.

SEM images of the L. gmelinii sample bodies before and after extraction were acquired. The images in Figure 4 reveal the structural changes in the samples. Compared with the untreated material (Figure 4(a)), the HWE processed 


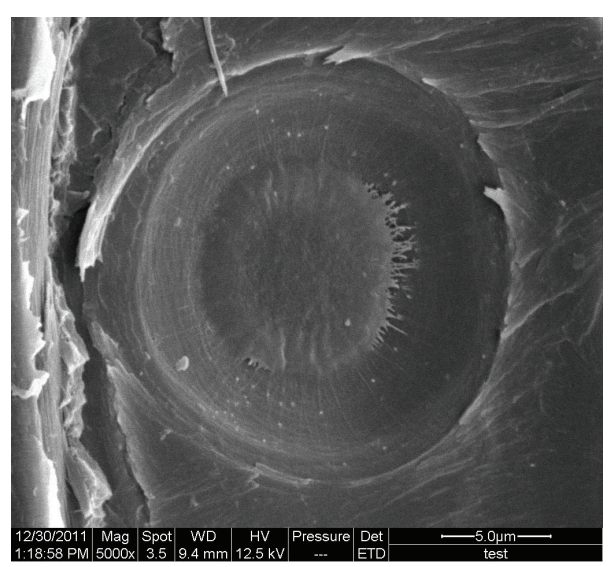

(a)

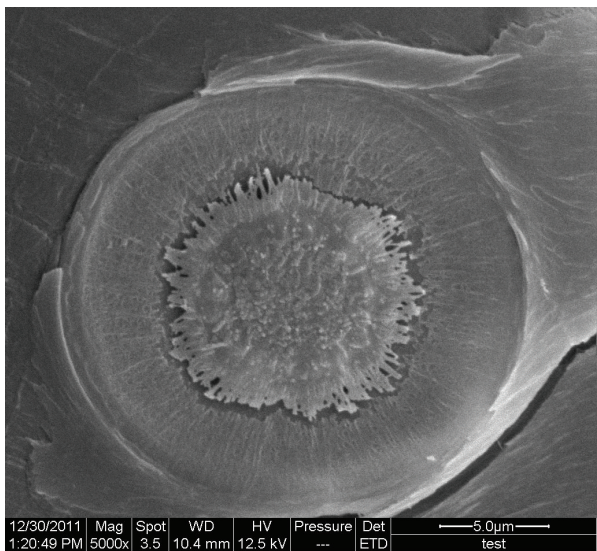

(b)

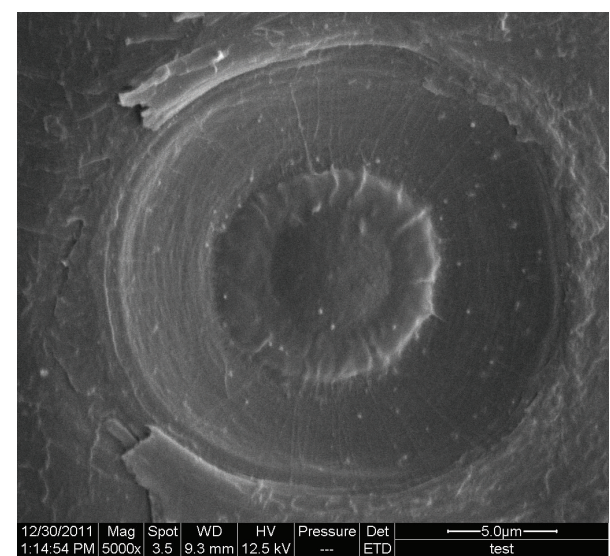

(c)

FIgURE 4: SEM images L. gmelinii. (a) Untreated sample. (b) Sample after UAE. (c) Sample after HWE.

material (Figure 4(c)) shows that the grain hole and the texture of L. gmelinii sample bodies were not completely processed. The UAE processed material (Figure 4(b)) reveals a more significant impact caused by the ultrasonic cavitation. A spongy structure with a defined fracture on the L. gmelinii sample bodies can also be seen more clearly, suggesting that ultrasonic vibration also increased the mass transfer rates of the target compounds from the material to the water, thus producing a higher extraction yield of the arabinogalactan.

\section{Conclusions}

In this work, we optimized the ultrasound-assisted extraction process of arabinogalactan from dihydroquercetin-extracted residues. The UAE conditions were optimized in detail and were found to be an extraction temperature of $41.5^{\circ} \mathrm{C}$, an extraction time of $24.3 \mathrm{~min}$, and a liquid-solid ratio of 40 $(\mathrm{mL} / \mathrm{g})$. Under these optimal conditions, the highest arabinogalactan extraction yield obtained was $11.18 \%$. Compared with the traditional $\mathrm{HWE}$ conditions (liquid-solid ratio $10 \mathrm{~mL} / \mathrm{g}, 50^{\circ} \mathrm{C}$ extraction temperature, and 120 -min extraction time), the UAE approach provided a higher extraction yield (9.53\% for HWE) and shorter extraction time. UAE may also be a time-saving method for the extraction of polysaccharides from other plant materials. The arabinogalactan had moderate antioxidant activity, although much less than that of vitamin C. FTIR spectra demonstrated that the basic physicochemical aspects of the extracted arabinogalactan from both procedures were similar to the pure material. SEM structural images before and after extraction revealed that UAE is more efficient for arabinogalactan extraction than HWE. In summary, an efficient method has been developed for the extraction of arabinogalactan from L. gmelinii, and it also shows great promise for the extraction and the separation of arabinogalactan from other plant materials as well.

\section{Acknowledgments}

The authors would like to thank the Special Fund for Forestry Scientific Research in the Public Interest (201304601), the National Natural Science Foundation of China (NSFC31170575), and the Natural Science Foundation of Heilongjiang Province of China (C201114) for their financial support.

\section{References}

[1] L. Yang, X. Sun, F. Yang, C. Zhao, L. Zhang, and Y. Zu, "Application of ionic liquids in the microwave-assisted extraction 
of proanthocyanidins from larix gmelini bark," International Journal of Molecular Sciences, vol. 13, no. 4, pp. 5163-5178, 2012.

[2] Y. Wang, Y. Zu, J. Long et al., "Enzymatic water extraction of taxifolin from wood sawdust of Larix gmelini (Rupr.) Rupr. and evaluation of its antioxidant activity," Food Chemistry, vol. 126, no. 3, pp. 1178-1185, 2011.

[3] L. Yang, J.-M. Huang, Y.-G. Zu et al., "Preparation and radical scavenging activities of polymeric procyanidins nanoparticles by a supercritical antisolvent (SAS) process," Food Chemistry, vol. 128, no. 4, pp. 1152-1159, 2011.

[4] Y. Wang and S. Wang, "Extraction of dihydroquercetin in larch," Food Science, vol. 30, no. 24, pp. 141-143, 2009.

[5] L. Yang, C. Ma, J. Huang, Z. Sun, T. Liu, and Y. Zu, "Comparison of dihyroquercetin extraction methods from Larix gmelinii," Forest Engineering, vol. 25, no. 9, pp. 6-10, 2009.

[6] C. Ma, Z. Sun, J. Huang, T. Liu, L. Yang, and Y. Zu, "Extraction of dihydroquercetin from larch wood with ultrasonic-microwave alternant method," Chemical Industry and Engineering Progress, vol. 29, no. 1, pp. 134-145, 2010.

[7] E. N. Medvedeva, V. A. Babkin, and L. A. Ostroukhova, "Arabinogalactan from larch-properties and usage perspectives (review)," Chemical Plant Stock, vol. 1, no. 1, pp. 27-37, 2003.

[8] A. M. Svetlana, P. A. Galina, I. D. Valentina et al., "Larch arabinogalactan as a perspective polymeric matrix for biogenic metals," Chemistry and Computational Simulation Butlerov Communications, vol. 2, no. 7, pp. 47-50, 2002.

[9] E. V. Groman and D. Gou, "Development of an immunoassay for larch arabinogalactan and its use in the detection of larch arabinogalactan in rat blood," Carbohydrate Research, vol. 301, no. 1-2, pp. 69-76, 1997.

[10] E. M. Goellner, J. Utermoehlen, R. Kramer, and B. Classen, "Structure of arabinogalactan from Larix laricina and its reactivity with antibodies directed against type-IIarabinogalactans," Carbohydrate Polymers, vol. 86, no. 4, pp. 1739-1744, 2011.

[11] R. Marett and J. L. Slavin, "No long-term benefits of supplementation with arabinogalactan on serum lipids and glucose," Journal of the American Dietetic Association, vol. 104, no. 4, pp. 636-639, 2004.

[12] L. Josephson, E. V. Groman, C. Jung, and J. M. Lewis, “Targeting of therapeutic agents using polysaccharides," US Patent 5.336.506.

[13] Y. S. Ovodov, "Polysaccharides of phanerogams: their structure and physiological activity," Russian Journal of Bioorganic Chemistry, vol. 24, no. 7, pp. 483-501, 1998.

[14] N. L. Currier, D. Lejtenyi, and S. C. Miller, "Effect over time of in-vivo administration of the polysaccharide arabinogalactan on immune and hemopoietic cell lineages in murine spleen and bone marrow," Phytomedicine, vol. 10, no. 2-3, pp. 145-153, 2003.

[15] G. S. Kelly, "Larch arabinogalactan: clinical relevance of a novel immune-enhancing polysaccharide," Alternative Medicine Review, vol. 4, no. 2, pp. 96-103, 1999.

[16] I. M. Borisov, E. N. Shirokova, V. A. Babkin, G. A. Tolstikov, and Y. B. Monakov, "Mechanism of the peroxide oxidation of arabinogalactan in a water-based medium," Doklady Chemistry, vol. 383, no. 4-6, pp. 117-119, 2002.

[17] R. I. Pinhassi, Y. G. Assaraf, S. Farber et al., "Arabinogalactanfolic acid-drug conjugate for targeted delivery and targetactivated release of anticancer drugs to folate receptoroverexpressing cells," Biomacromolecules, vol. 11, no. 1, pp. 294$303,2010$.
[18] T. G. Tolstikova, M. V. Khvostov, A. O. Bryzgalov, A. V. Dushkin, and G. A. Tolstikov, "Arabinogalactan, a plant polysaccharide, as a new tool for pharmacon clathration," Doklady Biological Sciences, vol. 433, no. 1, pp. 247-248, 2010.

[19] B. T. Kusema, C. Xu, P. Mäki-Arvela et al., "Kinetics of acid hydrolysis of arabinogalactans," International Journal of Chemical Reactor Engineering, vol. 8, article A44, 2010.

[20] B. T. Kusema, G. Hilmann, P. Mäki-Arvela et al., "Selective hydrolysis of arabinogalactan into arabinose and galactose over heterogeneous catalysts," Catalysis Letters, vol. 141, no. 3, pp. 408-412, 2011.

[21] C.-H. Ma, T.-T. Liu, L. Yang, Y.-G. Zu, S.-Y. Wang, and R.R. Zhang, "Study on ionic liquid-based ultrasonic-assisted extraction of biphenyl cyclooctene lignans from the fruit of Schisandra chinensis Baill," Analytica Chimica Acta, vol. 689, no. 1, pp. 110-116, 2011.

[22] L. Yang, H. Wang, Y.-G. Zu et al., "Ultrasound-assisted extraction of the three terpenoid indole alkaloids vindoline, catharanthine and vinblastine from Catharanthus roseus using ionic liquid aqueous solutions," Chemical Engineering Journal, vol. 172, no. 2-3, pp. 705-712, 2011.

[23] L. Yang, Y. Liu, Y.-G. Zu et al., "Optimize the process of ionic liquid-based ultrasonic-assisted extraction of aesculin and aesculetin from Cortex fraxini by response surface methodology," Chemical Engineering Journal, vol. 175, no. 1, pp. 539-547, 2011.

[24] Y. Zuo, L. Zhang, J. Wu, J. W. Fritz, S. Medeiros, and C. Rego, "Ultrasonic extraction and capillary gas chromatography determination of nicotine in pharmaceutical formulations," Analytica Chimica Acta, vol. 526, no. 1, pp. 35-39, 2004.

[25] Y. Jiao and Y. Zuo, "Ultrasonic extraction and HPLC determination of anthraquinones, aloe-emodine, emodine, rheine, chrysophanol and physcione, in roots of Polygoni multiflori," Phytochemical Analysis, vol. 20, no. 4, pp. 272-278, 2009.

[26] C. Wang and Y. Zuo, "Ultrasound-assisted hydrolysis and gas chromatography-mass spectrometric determination of phenolic compounds in cranberry products," Food Chemistry, vol.128, no. 2, pp. 562-568, 2011.

[27] M. Dubois, K. A. Gilles, J. K. Hamilton, P. A. Rebers, and F. Smith, "Colorimetric method for determination of sugars and related substances," Analytical Chemistry, vol. 28, no. 3, pp. 350356, 1956.

[28] H. Wang, X. D. Gao, G. C. Zhou, L. Cai, and W. B. Yao, "In vitro and in vivo antioxidant activity of aqueous extract from Choerospondias axillaris fruit," Food Chemistry, vol. 106, no. 3, pp. 888-895, 2008.

[29] H.-B. Li, C.-C. Wong, K.-W. Cheng, and F. Chen, "Antioxidant properties in vitro and total phenolic contents in methanol extracts from medicinal plants," LWT-Food Science and Technology, vol. 41, no. 3, pp. 385-390, 2008.

[30] H. V. Kumar, C. R. Gnanendra, N. Naik, and D. C. Gowda, "In vitro antioxidant activity of dibenz[b,f]azepine and its analogues," E-Journal of Chemistry, vol. 5, no. 2, pp. 1123-1132, 2008.

[31] S. D. Katekhaye and M. S. Kale, "Antioxidant and free radical scavenging activity of Pithecellobium dulce (Roxb.) Benth wood bark and leaves," Free Radicals and Antioxidants, vol. 2, no. 3, pp. 47-57, 2012.

[32] P. S. Negi, A. S. Chauhan, G. A. Sadia, Y. S. Rohinishree, and R. S. Ramteke, "Antioxidant and antibacterial activities of various seabuckthorn (Hippophae rhamnoides L.) seed extracts," Food Chemistry, vol. 92, no. 1, pp. 119-124, 2005. 

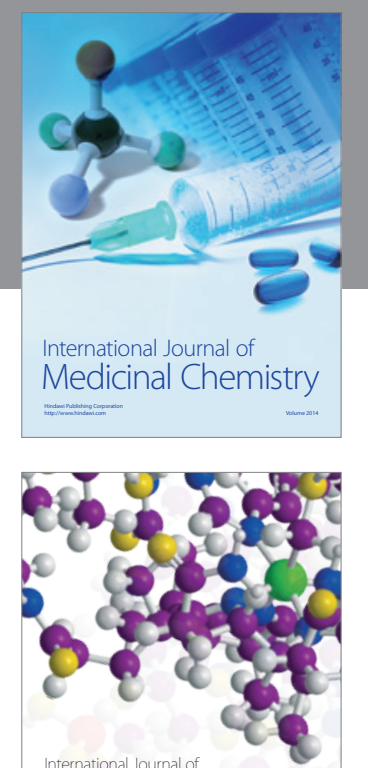

\section{Carbohydrate} Chemistry

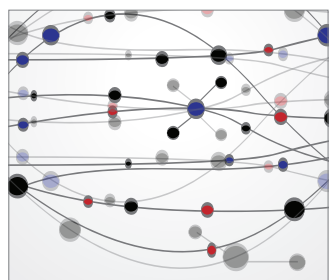

The Scientific World Journal
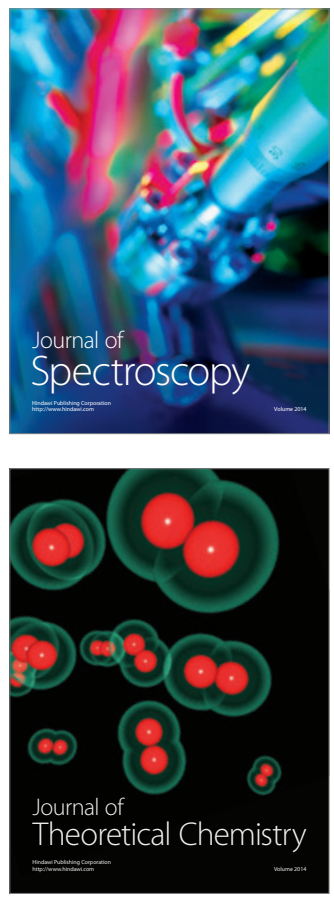
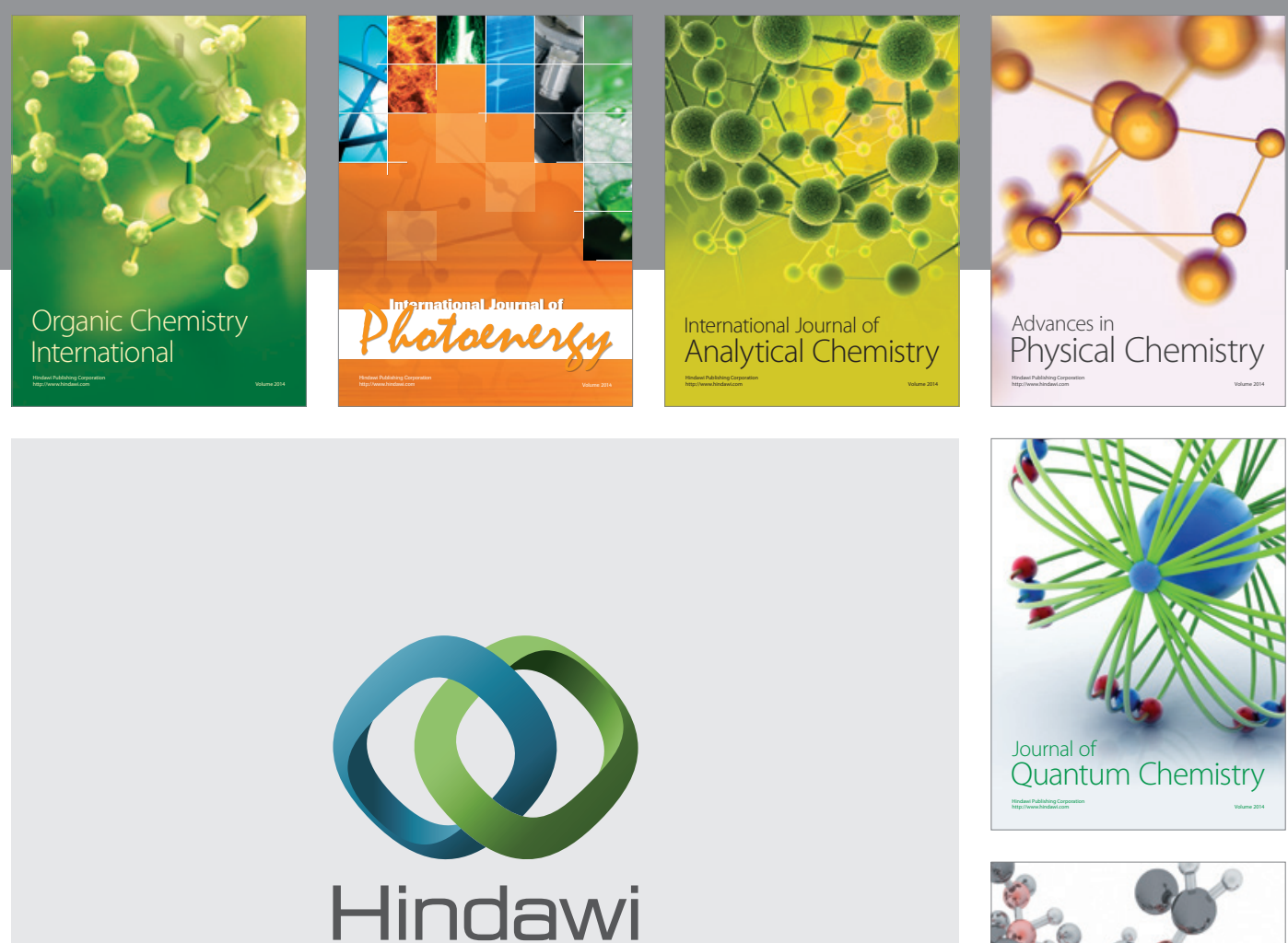

Submit your manuscripts at

http://www.hindawi.com

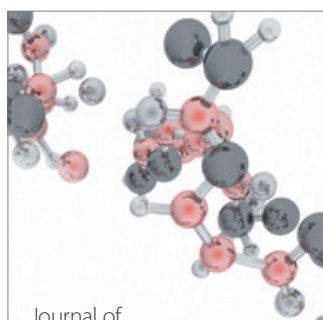

Analytical Methods

in Chemistry

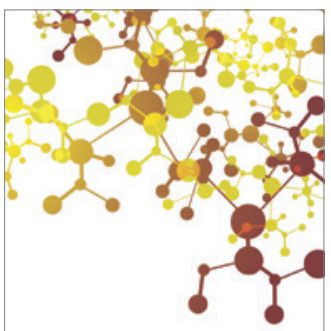

Journal of

Applied Chemistry

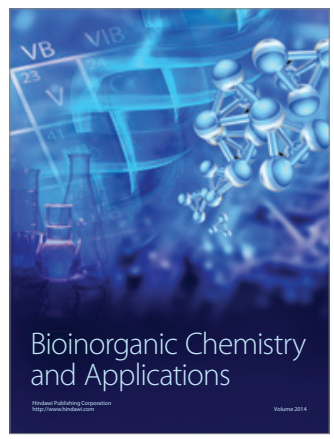

Inorganic Chemistry
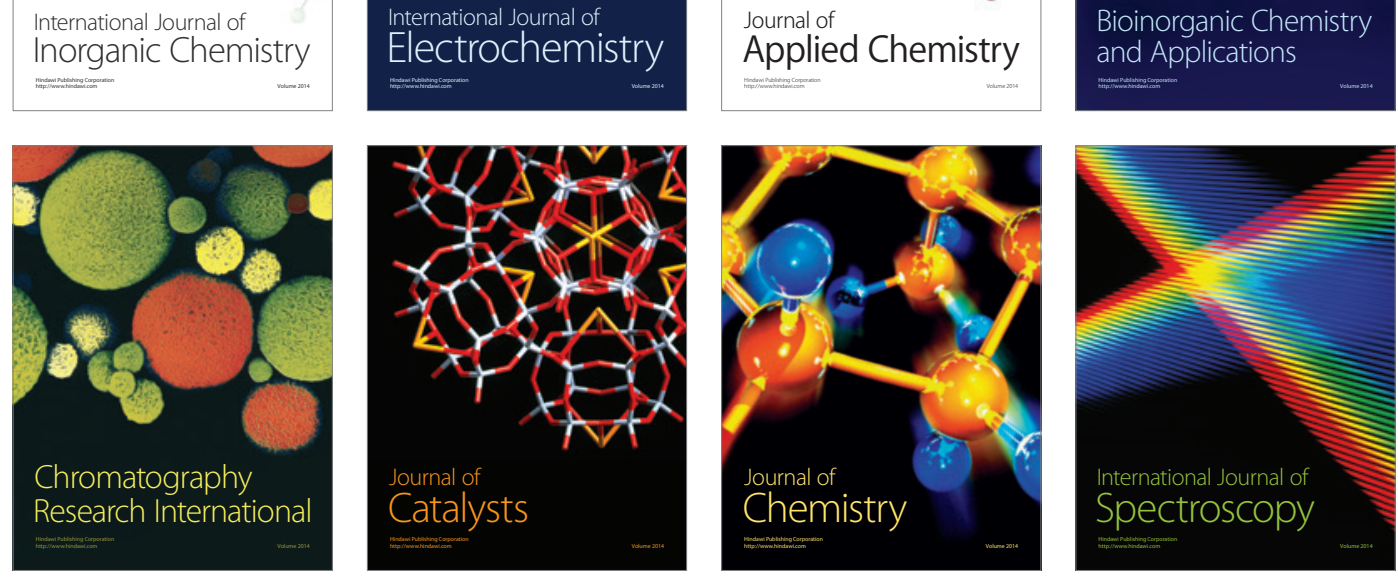